\title{
Precision Determination of Atomic Masses
}

\author{
A. H. Wapstra, Delft and Amsterdam
}

(University of Technology, Delft and Institut voor Kernphysich Onderzoek)

Recently, the first report was published of an experiment ${ }^{1}$ ) in which the charge of a pion was changed by two units. The oxygen isotope ${ }^{18} \mathrm{O}$ was bombarded with positive pions, which changed pairs of neutrons in the nucleus into protons and emerged as negative pions :

$$
{ }^{18} \mathrm{O}+\pi+=\pi-+{ }^{18} \mathrm{Ne}
$$

Observation of this reaction is difficult because of its very small cross section. Its existence could only be confirmed because a peak was seen at a negative pion energy corresponding to an energy balance involving the known atomic masses of ${ }^{18} \mathrm{O}$ and ${ }^{18} \mathrm{Ne}$ in their ground states.

This reaction is only one of very many types of reaction between nuclear and elementary particles, and in each of them such an energy balance is important. One point that should be made clear at the beginning is that the quantities determined most accurately by experiments, and those most useful in dayly use, are indeed atomic masses and not nuclear ones. The difference between the two quanties is the weight of all electrons around the nucleus decreased by that of their binding energy to the nucleus. The last quantity, at most equivalent to 3 three parts per million of the nuclear mass, is only known to an accuracy far worse than that with which atomic masses can be determined. Consider the $\alpha$-decay :

$$
{ }^{210} \mathrm{Po}(\alpha) \rightarrow{ }^{206} \mathrm{~Pb}
$$

The energy $E_{\alpha}$ of the $\alpha$-particle $\left({ }^{4} \mathrm{He}\right)$ plus that of the recoiling ${ }^{206} \mathrm{~Pb}$ nucleus (which is approximately 4/206) $E_{\alpha}$ should be very nearly equal to the energy equivalent of the atomic mass difference

$$
M\left({ }^{210} \mathrm{Po}\right)-M\left({ }^{206}\right) \mathrm{Pb}-M\left({ }^{4} \mathrm{He}\right) \text {. }
$$

To see this, we first notice that the mass of all electrons involved is taken into account. The charge of the nucleus decreases by two units, being the charge of the $\alpha$-particle; thus the neutral ${ }^{206} \mathrm{~Pb}$ atom has two electrons less than the ${ }^{210} \mathrm{Po}$ atom, but these appear again in the mass of ${ }^{4} \mathrm{He}$. As to the balance of electron binding energies, a small (sufficiently well known) correction has to be made for that in ${ }^{4} \mathrm{He}$, considering that the particle emerging is the ion ${ }^{4} \mathrm{He}^{++}$, a bare nucleus. The main point is, however, the difference in electron binding energies in ${ }^{210} \mathrm{Po}$ and ${ }^{206} \mathrm{~Pb}$. This quantity, about $30 \mathrm{keV}$, is known far less precisely than the energy of the $\alpha$-particles emitted in this process which is known to a precision of $0.09 \mathrm{keV}$. Now, the binding energy of the electrons in ${ }^{210} \mathrm{Po}$ is larger than that in ${ }^{206} \mathrm{~Pb}$. Thus, energy has to be added to the electron system in the decay process, which means that the $\alpha$-particle if departing with an energy defined by expression 1), but taking nuclear instead of atomic masses, would leave ${ }^{206} \mathrm{~Pb}$ behind in a state lower than its ground state, which is of course impossible. The $\alpha$-particle thus has to increase the total energy of the atomic electrons during its passage through the electron shells. A similar situation exists for nuclear reactions initiated by particles with energies not higher than a few tens of $\mathrm{MeV}$. Thus generally speaking, the numbers of interest for nuclear physics are not nuclear but atomic masses.

For this reason, it is fortunate that the only method available for absolute measurements (in contrast to the mass differences obtained in nuclear reactions), mass spectroscopy also measures atomic rather than nuclear masses. The reason is that these measurements are made by studying the influence of magnetic and/or electric fields on the orbits of ions obtained by removing one or two outer electrons from the atoms. Thus, atomic masses can be derived by making a small and sufficiently well known correction to the ion masses measured (though the precision of mass spectrometers is nearing the stage that significant new information on the electron binding energies can be obtained from such measurements).

The best mass spectrometer built so far was designed and constructed by Lincoln G. Smith ${ }^{2}$ ) in Princeton. After his untimely death, it was transported to Delft Technological University where it has been reconstructed. It is now in the process of being tested. Its properties are described below.

\section{Mass Spectrometers}

The order of magnitude of the precision required is about 1 in $10^{\circ}$ (that is about $0.2 \mathrm{keV}$ for ${ }^{210} \mathrm{Po}$ compared to the $0.09 \mathrm{keV}$ precision mentioned above of its $\alpha$-particle energy). All precision mass spectrometers in use are based on the action of a known magnetic field on the paths of ions. The present, and indeed the foreseeable. technology does not allow the magnetic field along these paths to be determined with the necessary accuracy. For this reason, all mass spectroscopic techniques compare the action of the magnetic field on the paths of two ion beans coinciding as nearly as possible (mass doublets). Examples of such doublets are :

$$
\begin{gathered}
{ }^{200} \mathrm{Hg}^{++} ;\left({ }^{12} \mathrm{C}_{7}{ }^{1} \mathrm{H}_{16}\right)^{+} \\
\left({ }^{12} \mathrm{C}_{9}{ }^{1} \mathrm{H}_{20}\right)^{+} ;\left({ }^{12} \mathrm{C}_{10}{ }^{1} \mathrm{H}_{8}\right)^{+}
\end{gathered}
$$

Evidently, care is taken that the sum of the mass numbers in the two ion beams divided by their charge number is the same. The relative mass difference is then at most of the order of one part in a thousand of the sum of the mass numbers; thus, this difference need only be measured with a precision of one part in a million or less.

One might at this stage object that no absolute masses are measured. One should remember, though, that the unit for atomic masses is $1 / 12$ of the mass of the ${ }^{12} \mathrm{C}$ atom in its (atomic and nuclear) ground state. Thus, the doublet $b)$ equals $12 \times\left\{M\left({ }^{\prime} H\right)-1\right\}$ and measures the mass of the hydrogen atom, a very fundamental natural constant. And with its help, doublet a) gives indeed a value for the absolute mass of ${ }^{200} \mathrm{Hg}$.

Even so, the orbits of the two ion beams might be sufficiently different to cause systematic errors due to inhomogeneities in the magnetic field, if the two ion beams were accelerated to the same energy (or, what is the same, by the same voltage) before entering the magnetic field. Therefore, the beams are accelerated with voltages inversely proportional to the masses of the ions to be compared. One can even use the magnetic field as zero indicator and determine the mass ratio by measuring the required voltage ratio. In the Delft RF spectrometer a different method is used as described below.

The succes of such techniques is limited by the fact that unintended 


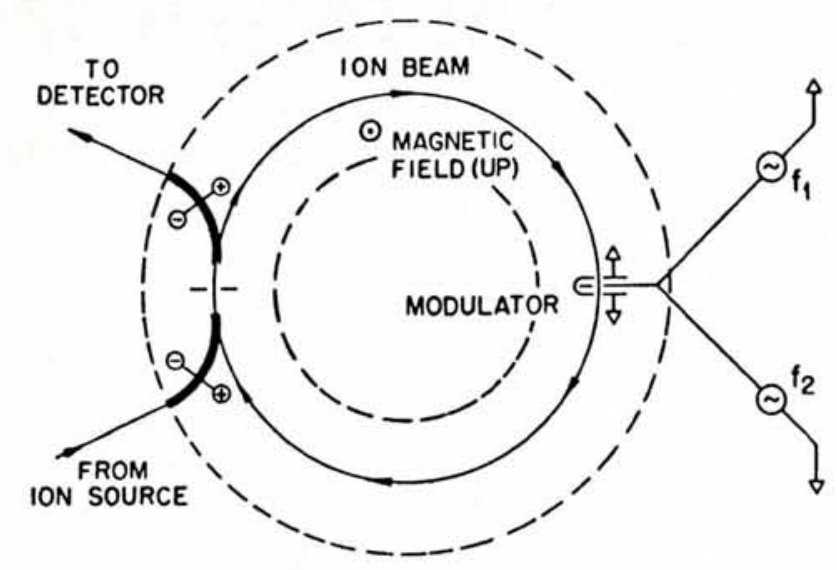

Fig. 1. Plan and elevation of the part of the instrument in the magnetic field.

vals of the RF oscillators. In those intervals though, the radial deviation at $360^{\circ}$ past injection is a maximum. Thus, the tails can be suppressed by mounting a "phase defining slit " at this position (see Fig. 1).

The instrument actually does not contain input and exit slits in the magnetic field. In their stead, symmetric electric toroidal lens systems (which can be seen in Fig. 2) form images of slits at their other ends in such a way that the overall resolution is increased by a factor six.

The entrance slit of the first lens system is fed by the two-ion beam. The method of comparing the two components is, essentially, the following. The two components are admitted alternately, at a rate of 15 times per second. In each $30 \mathrm{~ms}$ time interval, the radio-frequency is swept through a frequency interval around the resonance of the beam considered. These

electrical fields (due, e.g. to surface charges on the inside of vacuum boxes) act differently on the two beams and thus may again cause systematic errors. Such errors are especially severe in wide doublets, e.g. if the sums of the mass numbers does not match, as in :

$$
\left({ }^{12} \mathrm{C}_{9}{ }^{1} \mathrm{H}_{20}\right)^{+} ;\left({ }^{12} \mathrm{C}_{9}{ }^{1} \mathrm{H}_{19}\right)^{+}
$$

Indeed, deviating values have often been found for such wide doublets. One can, though, use such non-closing doublets for estimating the effect of beam shifts. Essentially, this means that the real measurement is that of a ratio of mass differences, such as:

$\left(\mathrm{C}_{9} \mathrm{H}_{20}-\mathrm{C}_{10} \mathrm{H}_{8}\right) /\left(\mathrm{C}_{9} \mathrm{H}_{20}-\mathrm{C}_{9} \mathrm{H}_{19}\right)$.

The second mass difference, practically the mass of the hydrogen atom, is known to one part in $10^{8}$. No error of any significance is therefore introduced by multiplying this ratio with that mass difference, and this is commonly done.

\section{Lincoln Smith's RF Mass Spectrometer}

Lincoln Smith's instrument is based on the knowledge that the revolution frequency $v_{\mathrm{c}}$ of an ion with mass $M$ and charge $Q$ on its circular path in a homogeneous magnetic field $B$ is, in non-relativistic approximation:

$$
v_{\mathrm{c}}=Q B / 2 \pi M
$$

independent of its position and practically of its energy. (The relativistic corrections are small, but not negligible at the required level of precision, for ion beams of $10-30 \mathrm{keV}$.) Thus, an instrument based on this principle is double focusing as defined by Mattauch (that is, independent of beam energy and direction, to a certain limit.)

The precision of any spectrometer is the product of the relative line width (reverse resolution) and the relative precision with which the line position can be determined. In order to reach high resolution, Smith proposed the following method (see Fig. 1). The ion beams are inflected in the magnetic field through an entrance slit, at a pitch of $1 \%$. They thus describe a narrow spiral. After $180^{\circ}$ and $540^{\circ}$, they are focused on to modulator slits. If no voltage occurs across these modulators, the beam is finally focussed on to an exit slit and and deflected in a way symmetric to the inflection.

If the ion beam, on passing through the modulator, is accelerated or decelerated, it misses the exit slit, except when the acceleration in the second modulator is the exact opposite of that in the first. This can be made to occur continuously if the modulators are fed with a frequency $v=(\mathrm{n}+1 / 2) v_{\mathrm{c}}$ $n$ being an integer.

The resolution depends on the accelerating voltage and is proportional to $\mathrm{n}$. With a voltage of about $100 \mathrm{~V}$ and $n \sim 1000$, a resolution of about 500000 has been obtained.

The resolution could be worsened by long tails due to the fact that, slightly off resonance, the accelerating and decelerating voltages still match for considerable phase interfrequency intervals are adjusted in such a way that the two spectral lines, depicted on an oscilloscope screen, coincide. In view of the requirement that the paths through the magnetic field of the two ion beams should coincide as well as possible, all relevant voltages are switched proportionally to the frequencies.

The precision in measuring the frequency settings of the lines has been determined by comparing an ion beam with itself, a sufficient number of times. Plotting the observed relative positions, it was found that these positions can be determined in one measurement with a precision of one part in 2500 of the line width ${ }^{3}$ ). Multiplication with the resolution given above shows that the desired precision of 1 in $10^{\circ}$ has been reached with this technique.

The question is, though, whether no systematic errors occur. As is clear from the above description, Smith took great care to avoid such errors as far as possible. He did even more: all critical parts within the vacuum system were gilded in order to keep them as clean as possible, to avoid surface charges. (Strangely enough, this may not have been the best way: coating with aquadag may be more effective).

How can one check the resulting quality of the system ? In the first place, by checking wide doublets, as mentioned above. Smith found that the frequency ratio of such $\left({ }^{1} \mathrm{H}\right)$ wide doublets did not quite fit the known 
Fig. 2 Photograph of the spectrometer showing the toroidal lens system replacing the entrance and exit slits.

mass ratio. Since this was probably due to remaining electrical fields, Smith reasoned that he could compensate their effect by some other electric field generated on purpose; he did so by putting a voltage on the phase defining slit. In the beginning, this voltage had to be $1.5 \mathrm{~V}$, but after having made several improvements ${ }^{4}$ ) it could be reduced more than an order of magnitude.

A second kind of check is to compare the measured results for different doublets between which a known relation should exist, such as doublet $b$ above and the doublets :

$$
\begin{aligned}
& \mathrm{C}_{5}{ }^{2} \mathrm{D}_{12} ; \mathrm{C}_{6}{ }^{2} \mathrm{D}_{6} \\
& \mathrm{C}_{6} \mathrm{H}_{12} ; \mathrm{C}_{6} \mathrm{D}_{6}
\end{aligned}
$$

One will observe that $c+d-b=0$ Checking a somewhat extended set of such relations with statistical (least squares) methods gives a check on the reliability of the result.

Such a check depends on the errors assigned to the measured results. The common way of assigning errors to mass spectrometrical measurements is measuring each doublet repeatedly and assigning as error to each single measurement the observed statistical spread in the results (the final error in the reported average is then this number divided by the square root of the number of measurements). Smith made an extra check on this result by comparing it to the product of the relative precision of determining the position of a line (for which the value $1 / 2500$ was reported above) and the actual resolution in the measurement. Generally speaking, the two values agreed reasonnably well.

Strictly speaking, the error values thus derived give the reproducibility and not the accuracy of the measurement. It is, therefore, not surprising that the statistical analysis mentioned above indicates that the real errors are about a factor two larger. Even after multiplication of all reported errors with this correction factor, one can maintain that systematic errors of a somewhat sophisticated type may still influence the results. It would be desirable that a third check could be made by comparing these data with nuclear reaction results. The number of nuclear reaction energy measurements of comparable precision is, however, very limited. The class of reactions offering most possibilities contains $(n, \gamma)$ reactions, but at pre-

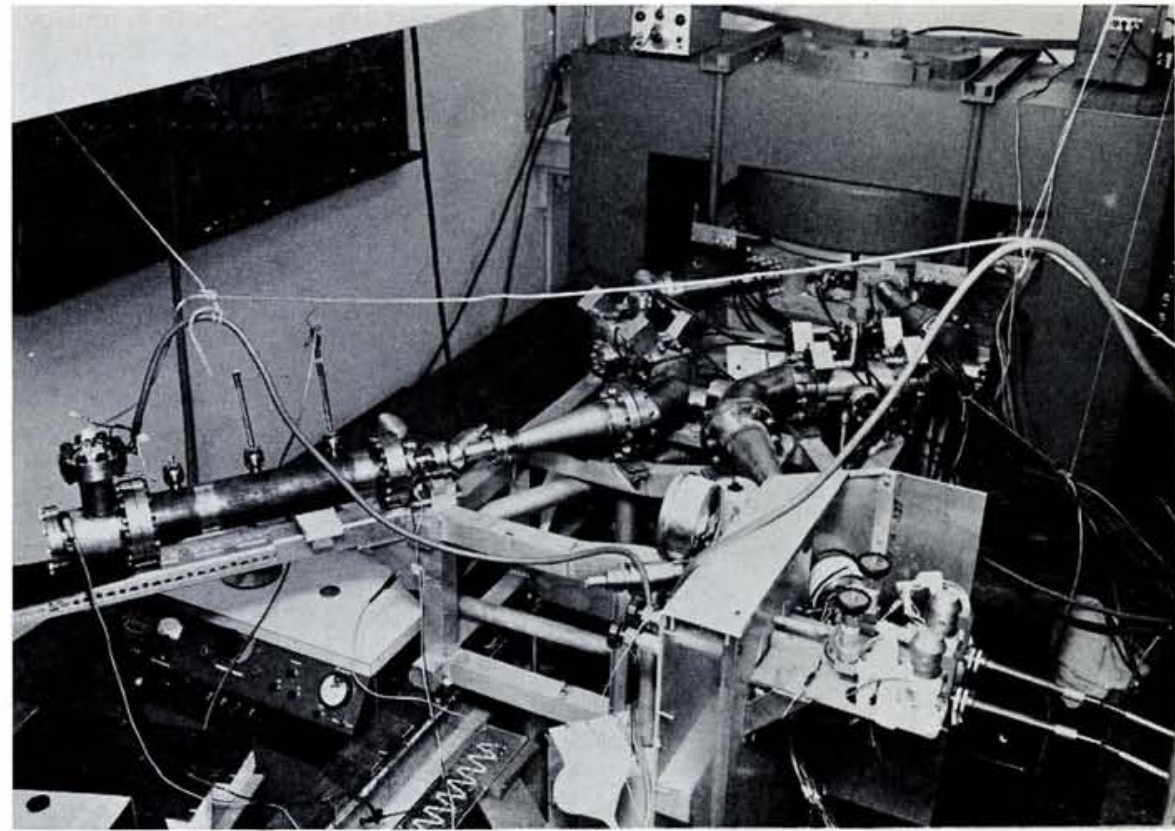

sent the best calibration for such reactions is derived from Smith's mass spectroscopic results ${ }^{4}$ ) and can therefore not be used as a check.

The only reaction energy now available for this purpose is Bergquists ${ }^{5}$ ) measurement of the beta decay energy in the reaction ${ }^{3} \mathrm{H} \rightarrow\left(\beta^{-}\right)+{ }^{3} \mathrm{He}$. Both his measurement and Lincoln Smith's measurement on the ions ${ }^{3} \mathrm{He}^{+}$and $\mathrm{H}^{3} \mathrm{H}^{+}$were so precise that corrections had to be made for electron binding energies. In making these corrections, Smith assumed that his ${ }^{3} \mathrm{He}^{+}$ions all had their remaining electron in the $1 \mathrm{~s}$ orbit. His result then deviated from Bergquist's by a few statistical errors, but in such a way that the difference would disappear if a sizeable fraction of the ${ }^{3} \mathrm{He}^{+}$ions were in the metastable 2 s state. On the other hand, these measurements were made before Smith made his last improvements on the spectrometer, which means that the difference could as well be due to the systematic errors that were then still present.

\section{Work in Delft}

The spectrometer has been moved to Delft and installed, and it has already been possible to reproduce with reasonable consistency some measurements done earlier in Princeton. In several respects, improvements have been made ${ }^{6}$ ). Special care has been taken to make the construction independent of vibrations. A study has been made of the harmonic purity of the radio-frequency as it will be clear that even harmonics will have undesirable consequences.

Unfortunately, the rather sophisticated electronics is by now somewhat old and not reliable. No money has yet been found to replace it by more modern and stable electronics, which would quite probably allow the resolution and accuracy to be improved by about an order of magnitude. This would not be very important for nuclear physics, but it might bring us to the border of the region where significant observations in atomic and molecular electron binding energies might be made.

Though not completely stable, the instrument is operational. A study has been made of its focusing properties ${ }^{6}$ ), with the telecentric object plane selector and magnifying projector developped in Delft by K.J.P. Stigter (see ref 7). This study has revealed that the measurements of Smith had probably been done with an improper beam. This may explain that in Smith's measurement the beam positions took a rather considerable time to stabilize. The proper beam, unfortunately, focuses as a cross, possibly due to improper alignment of the slits. Experiments are under way to correct this condition. We expect then an improved stability and a further reduction of the systematic errors.

Among the measurements to be made after curing this condition one may be mentioned because of its general physics interest. It concerns the definition of the mass unit, the $\mathrm{kg}$. Other units have by now been connected to natural constants, but the $\mathrm{kg}$ is still defined as the weight of a rather arbitrary piece of platinum-iridium in Paris. This standard can be compared with similarly constructed Pt-Ir standards with a precision of somewhat better than I part in $10^{\circ}$, but to other arbitrary masses with an accuracy somewhat less than that. Thus, if : 
a) one could make a crystal of a pure nuclide, in which the number of atoms were known to a higher precision than I part in $10^{\circ}$,

b) the atomic mass of this nuclide were also known to at least this precision,

one could base the unit of mass on that of the atomic mass unit defined above, $M\left({ }^{12} \mathrm{C}\right) / 12$ by defining a value for Avogadro's constant.

Collaborators at the National Bureau of Standards in Washington have high hopes that they could, given sufficient funding, fabricate crystals of $99,9999 \%$ pure silicon (with known admixtures of the other two stable isotopes, ${ }^{29} \mathrm{Si}$ and ${ }^{30} \mathrm{Si}$ ) which have fewer crystal faults than 1 in $10^{\circ}$. Then, if the atomic mass of ${ }^{28} \mathrm{Si}$ were known to the same precision (those of the other two isotopes need then be far less accurately known), one could start considering a mass definition along the indicated lines.

The present ${ }^{8}$ ) mass value of ${ }^{28} \mathrm{Si}$ is : $M\left({ }^{28} \mathrm{Si}\right)=27.9769281 \pm 0.0000007$ which is at least three times less accurate than 1 in $10^{\circ}$. Moreover, the determination is very indirect : the reported accuracy of the best available direct measurements ${ }^{9}$ ) is only 14 parts in $10^{9}$. In my opinion, both reported accuracies are overestimated by at least a factor three. We hope to apply the Delft RF mass spectrometer for a satisfactory determination of this atomic weight.

\section{REFERENCES}

1. MARKS, T., et al., Phys. Rev. Lett. 38 (1977) 149

2. SMITH, L.G., Proceedings of the Third International Conference on Nuclidic Masses, R.C. Barber (ed), (University of Manitoba Press) 1967, p. 811

3. SMITH, L.G., Phys. Rev. C4 (1971) 22

4. SMITH, L.G. and WAPSTRA, A.H., Phys. Rev. C11 (1975) 1392

5. BERGQUIST, K.E., Nucl. Phys. B39 (1972) 371

6. NAKABUSHI, $\mathrm{H}$. and KOETS, E., Mass Spectroscopy 25 (1977) 49

7. KOETS, E., Proceedings of the Fifth International Conference on Atomic Masses and Fundamental Constants, J.H. Sanders and A.H. Wapstra (ed), (Plenum Press) 1976, p. 164

8. WAPSTRA, A.H. and BOS, K., Atomic Data and Nuclear Data 19 (1977) 153

9. SCOLMAN, T.T., QUISENBERRY, K.S. and NIER, A.O., Phys. Rev. 102 (1956) 1976

\section{9th European Conference on Atomic Spectroscopy}

The annual conference of EGAS, the Atomic Spectroscopy Section of the Atomic Physics Division, arranged this year by D. Kunisz and her coworkers at the Jagellonian University, Cracow, attracted more than 230 participants from 16 countries among which several east European countries were well represented. The traditional style of EGAS meetings of having a small number of invited talks plus many contributed papers was again followed and, as always, young atomic physicists were encouraged to take the floor.

Laser spectroscopy continues to play a dominant role at EGAS meetings, with several important developments being reviewed in Cracow. Methods based on laser fluorescence today can detect concentrations as low as $10^{-2}$ atom $/ \mathrm{cm}^{3}$. Recent studies of hyperfine structure and isotope shifts for radioactive sodium isotopes ${ }^{26},{ }^{27},{ }^{28} \mathrm{Na}$ by laser methods were summarized by $P$. Jacquinot. E.-W. Otten and collaborators have used laser spectroscopy to measure isotope shifts in radioactive $\mathrm{Hg}$ nuclei, produced in the ISOLDE on-line mass separator, CERN. Very abrupt changes in charge radii around $A=184$ are explained in terms of "coexistence" of two different nuclear shapes which are energetically almost degenerate. Laser methods can thus be successfully applied to study nuclear properties. It seems clear that the technique of exciting atomic or fast ion beams from accelerators with laser light offers fascinating possibilites for future work.

One of the first atomic physics techniques utilizing accelerator beams, beam-foil spectroscopy is well suited to the measurement of radiative lifetimes in atoms and ions. A modified technique based on the spectroscopic study of sputtered, excited atoms has been developed in Aarhus by T. Andersen and his co-workers, and has given the level lifetimes in neutral uranium. Multiply-excited levels are strongly populated when ions interact with solid foils, and recent work from Aarhus shows that these states can also be studied by beam-gas techniques. The spectral resolution obtained in this way can be considerably higher than in traditional beam-foil spectroscopy.

Much interest is presently being focused on level lifetimes in atoms and ions and the related atomic transition probabilities, using laser methods, beam-foil technique, level crossing and intensity studies.

In spectral line classification and term analysis, perhaps the most complex spectra reviewed were those of $\mathrm{Np}$ I and $\mathrm{Np}$ II where angular momenta as high as $J=23 / 2$ have been observed at Orsay. One of the most "popular" elements among atomic spectroscopists is now Mo, many times ionized, because of its role as an impurity in high-temperature Tokamak plasmas.

In optical pumping experiments with alkali atoms, the polarization is usually preserved by using buffer gases such as $\mathrm{Ne}, \mathrm{Ar}, \mathrm{Xe}$. The interactions between alkali atoms and noble gas atoms contain many interesting problems, but agreement between theory and experiment is usually quite satisfactory. According to T. Skalinski, it would however, be useful to undertake relaxation experiments in larger magnetic fields as an additional test of present calculations.

Pressure broadening of spectral lines also attracts considerable interest. A quasimolecular theory based on the Franck-Condon principle is known to reproduce the line wings quite satisfactorily while the shapes of the line centres are better described by classical impact theory. Recently J. Szudy and W.E. Baylis have developed a unified theory which successfully combines the above-mentioned limited approaches. There are several applications and consequences of such work, e.g. in understanding pressure effects in doppler-free twophoton laser spectroscopy. A whole section of the Cracow meeting was devoted to pressure effects on line shapes which nowadays can be very accurately measured using laser excitation.

As a testing ground for quantum electrodynamics the bound system $\mu^{+} e^{-}$, muonium is superior to hydrogen because the proton form factor introduces uncertainties in the latter case. G. zu Putlitz discussed measurements of the hyperfine structure for the muonium ground state, recently concluded at LAMPF, the meson facility at Los Alamos. Observations of Zeeman transitions in a very high and uniform magnetic field have yielded the muonium hyperfine splitting and the magnetic moment of the muon with uncertainties as low as 0.3 and $1.4 \mathrm{ppm}$ respectively. The agreement with theory is excellent.

In addition to invited and contributed papers, the tradition of roundtable discussions was continued. The topics dealt with in Cracow were "Term analysis", "Laser spectroscopy", "Highly excited states in atoms" and "Atomic data for fusion".

Indrek Martinson 